\title{
Dynamic Light Scattering from Semidilute Actin Solutions: A Study of Hydrodynamic Screening, Filament Bending Stiffness and the Effect of Tropomyosin/Troponin-Binding
}

\author{
R. Götter, K. Kroy, E. Frey, M. Bärmann, and E. Sackmann \\ Department of Physics E22 (Biophysics Group), \\ Department of Physics T34 (Theoretical Physics), \\ Technische Universität München, \\ James-Franck-Strasse, D-85747 Garching, Germany.
}

(May 8, 2019)

\begin{abstract}
Quasi-elastic light scattering (QELS) is applied to investigate the effect of the tropomyosin/troponin complex $(\mathrm{Tm} / \mathrm{Tn})$ on the stiffness of actin filaments. The importance of hydrodynamic screening in semidilute solutions is demonstrated. A new concentration dependent expression for the dynamic structure factor $g(\boldsymbol{k}, t)$ of semiflexible polymers in semidilute solutions is used to analyze the experimental QELS data. A concentration independent value for the bending modulus $\kappa$ is thus obtained. It increases by $50 \%$ as a consequence of Tm/Tn binding in a 7:1:1 molar ratio of actin/Tm/Tn. In addition a new expression for the initial slope of the dynamic structure factor of a semiflexible polymer is used to determine the effective hydrodynamic diameter of the actin filament. Our results confirm the general relevance of the concept of (intrinsic) semiflexibility to polymer dynamics.
\end{abstract}

\section{INTRODUCTION}

The main motivation of the present work was to study the effect of tropomyosin/troponin (Tm/Tn) binding on the bending stiffness of actin filaments. The semiflexible filaments with a length of $400(? ?)$ bind along the groove between the two twisted strands of the actin filament (Figure 1). The binding is influenced by the presence of $\mathrm{Ca}^{++}$. This effect is of great biological significance, since it plays a central role in the regulation of the myosinactin coupling in muscles.

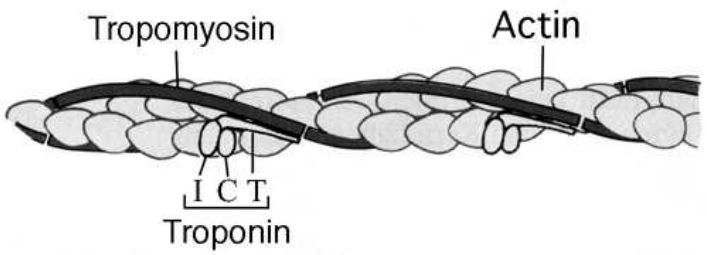

FIG. 1. Model of $\mathrm{Ca}^{++}$mediated Tm/Tn binding to actin filaments. Tm/Tn is known to bind along the groove of the actin filaments.

Actin, when polymerized in vitro, forms semiflexible macromolecules of contour lengths $L$ up to some $30 \mu \mathrm{m}$ and with a persistence length $L_{p}$ of at least some $\mu \mathrm{m}$. The system we are interested in is a semidilute solution of these macromolecules, i.e. an entangled network, where the single actin filaments interact strongly but the free volume is still much larger than the excluded volume. As a consequence of the great extension and large persistence length of the molecules, as compared to their lateral diameter $a$ (some $\mathrm{nm}$ ), the semidilute regime is unusually large. We probe the samples with scattering wavelengths $\lambda=2 \pi / k$ that are somewhat shorter than the mesh size $\xi$ of the network, which, in turn, is shorter than the persistence length $L_{p}$; i.e. the condition

$$
a \ll \lambda<\xi \ll L_{p}, L
$$

is fulfilled for all of our samples. An electron micrograph of a typical system under study is reproduced in Figure 2.

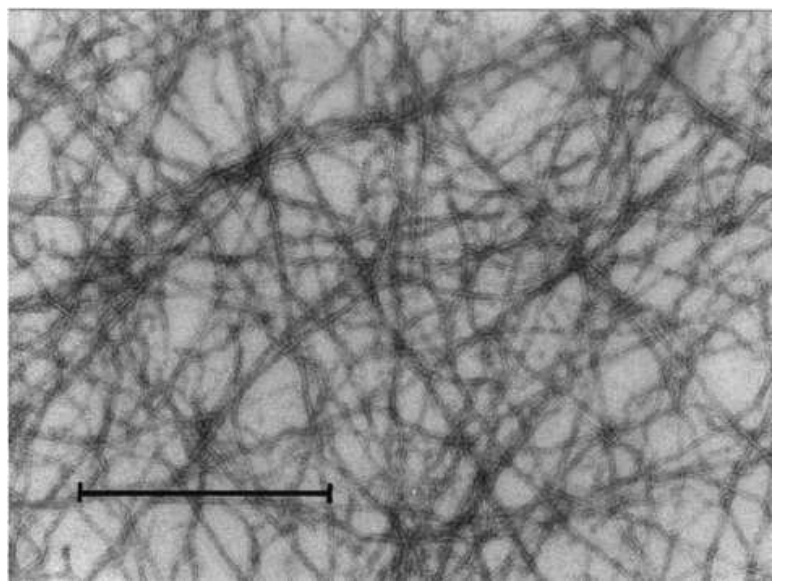

FIG. 2. Electron micrograph of a $0.4 \frac{\mathrm{mg}}{\mathrm{ml}}$ actin solution polymerized in vitro. The bar indicates the length of $1 \mu \mathrm{m}$. We probe the samples with scattering wavelengths $\lambda=2 \pi / k$ that are somewhat shorter than the mesh size $\xi$ of the network, which, in turn, is shorter than the persistence length $L_{p}$. The global motion of all polymers is strongly hindered by the entanglement.

The bending stiffness of a filament-like macromolecule may be inferred from an analysis of its conformational dynamics. Therefore various experimental techniques have been applied to investigate the dynamical properties of 
actin [1]. Solutions and gels were probed by high sensitivity rheology using torsional [2] and magnetic bead rheometers [3]. Käs et al. [4] and others [5] analyzed single labeled filaments by microfluorescence microscopy combined with dynamic image processing. There have also been several attempts to establish QELS as a quantitative method to probe the static and dynamical properties of semiflexible macromolecules (for critical discussions of the literature see e.g. Refs. [6] [8]). But in contrast to the dynamic properties of flexible polymers the dynamics of semiflexible polymers is not yet very well understood. This is mainly a consequence of the difficulties caused by the rigid constraint of a (virtually) unextensible contour. Models that try to represent the unextensible contour honestly [9, /7] have to deal with considerable technical difficulties. On the other hand, models that relax the constraint like the various modified versions of the so called Harris-Hearst-Beals-model [10] (for a short summary see [12]) allow for artificial stretching modes and predict a gaussian probability distribution for the spacial distances of the contour elements. As the most promising model with relaxed constraint we regard the model described in Refs. [11, 12, 8]. We will neither relax the constraint, nor do we need all the machinery developed by Aragón and Pecora in Refs. [9, ,7]. (However, one of our results, Eq. (2.8), may be obtained by their method [20].) In the special case, we are interested in, we profit from some simplifications brought about by the separation of length scales, Eq. (1.1), which in turn gives rise to a hierarchy of time scales: QELS measures the temporal decay of configurational correlations of the filaments. For long semiflexible actin filaments in entangled networks the internal configurational dynamics dominates over the center of mass and rotational motion of the molecules in time intervals typically probed by QELS. In addition, we can restrict ourselves to the weakly bending rod limit, where one can approximately take the undulations to be transverse to the mean contour [13,6], which is virtually fixed for the relevant time scales. The decay rate of the dynamic structure factor becomes thus accessible to a simple physical interpretation in terms of the bending modes only, i.e. the local bending modes may be studied "in isolation". This is a great practical advantage compared to the general case.

But the time decay of the structure factor also depends on the strength of hydrodynamic correlations. To obtain quantitative results it is therefore necessary to take into account the screening of the hydrodynamic selfinteraction of the filaments in semidilute solutions (or their finite length in the dilute case). As explained in Section II A this is most simply achieved by means of a concentration dependent renormalized friction coefficient, $\zeta_{\perp}$.

In contrast to the expression for the dynamic structure factor for intermediate times, which takes a simple form only after some approximations, its initial slope, resulting from the quasi-free Brownian fluctuations about the equilibrium configuration may be calculated exactly if Eq. (1.1) holds. The result is compared with previous experimental data in Section IIB. The good agreement of the theoretical prediction with the available data confirms our hypothesis that the intrinsic rodlike structure of polymers is a significant detail, which influences the dynamic properties. Moreover, the microscopic hydrodynamic diameter $a$ of the filament, which enters the dynamic structure factor as a parameter, also appears in the expression for the initial decay rate and may thus be determined by a measurement of the initial slope. Section III lists materials and methods, and the new experimental results are presented in Section IV. Finally we discuss possible refinements to our approach in Section $\mathrm{V}$.

\section{THEORETICAL BACKGROUND OF QELS FROM SEMIFLEXIBLE POLYMERS}

\section{A. Stretched Exponential Decay of the Dynamic Structure Factor}

The dynamic structure factor of a chain of length $L=N \Delta s$ with $N$ segments located at $\boldsymbol{r}_{n}(n=1, \ldots, N)$ is defined by

$$
g(\boldsymbol{k}, t)=\frac{1}{N} \sum_{n, m}\left\langle\exp \left[i \boldsymbol{k}\left(\boldsymbol{r}_{n}(t)-\boldsymbol{r}_{m}(0)\right)\right]\right\rangle .
$$

The brackets $\langle\ldots\rangle$ denote the ensemble average over all chain conformations, and $\boldsymbol{k}$ is the scattering wave vector. On the typical length scales probed by QELS $(0.1 \sim 1$ $\mu \mathrm{m})$ the conformational dynamics of actin filaments are dominated by the bending undulations. Consequently, we take the bending energy, given by the contour integral $\int_{L} d s$ over the local curvature multiplied by the bending modulus $\kappa$,

$$
E\left[\boldsymbol{r}_{s}\right]=\frac{\kappa}{2} \int_{L} d s\left(\frac{\partial^{2} \boldsymbol{r}}{\partial s^{2}}\right)^{2},
$$

to be the only relevant energetic term in a canonical description. This implies that the static mean square endto-end-distance is given by the well known Kratky-Porod formula 14 with the persistence length $L_{p}=\kappa / k_{B} T$. The time decay of the structure factor $g(\boldsymbol{k}, t) / g(\boldsymbol{k}, 0)$ is not easily calculated in the most general case. However, the particular case under study allows for simplifications, which enable us to adapt some of the ideas common in the theory of flexible polymers 15,16$]$. We start from the Langevin equation for a single polymer

$$
\frac{\partial}{\partial t} \boldsymbol{r}_{s}(t)=\int_{L} d s^{\prime} \boldsymbol{H}_{\perp}\left(\boldsymbol{r}_{s}, \boldsymbol{r}_{s^{\prime}}\right)\left(-\frac{\delta}{\delta \boldsymbol{r}_{s^{\prime}}} E\left[\boldsymbol{r}_{s^{\prime}}\right]+\boldsymbol{f}_{s^{\prime}}\right),
$$

where $\boldsymbol{f}_{s}$ is the stochastic force (white noise) and $\boldsymbol{H}_{\perp}$ is an effective mobility matrix, which takes into account the solvent-mediated self-interaction of the filament. This 
can be understood in analogy to the usual Oseen tensor. However, the effective reduction of the degrees of freedom due to the rigid constraint of a fixed contour length requires that the local longitudinal motion be projected out. In the weakly bending rod limit this is practically achieved by a suitable choice of coordinates: longitudinal distances are kept fixed, and the bending undulations are described by transverse coordinates (cf. Eq. (2.4) below). This is how one would address the problem in analogy to the classical Zimm model for flexible polymers, and how it was attempted previously in Ref. [17]. We shall neglect the center of mass and rotational motion, which are slow compared to the internal dynamics of the molecule as a consequence of the scale separation Eq. (1.1), the entanglement and hydrodynamic screening.

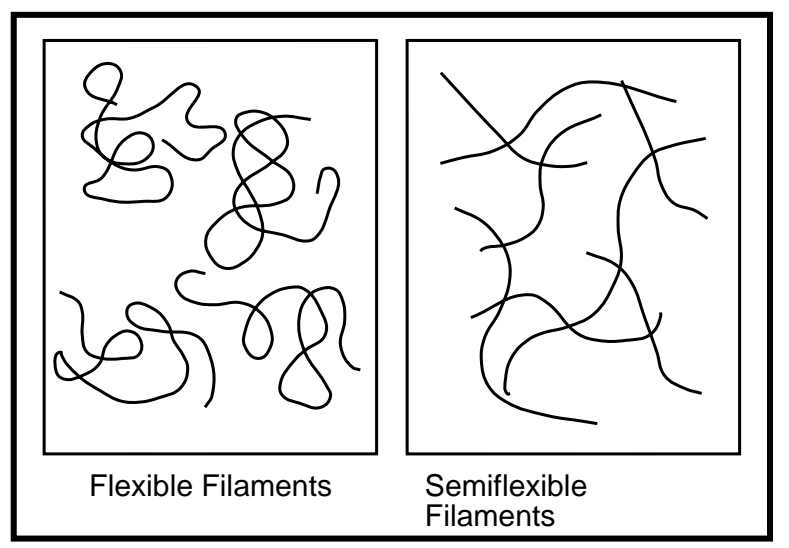

FIG. 3. Flexible filaments interact mostly with themselves, whereas semiflexible filaments interact with each other even at quite low concentrations. The perturbation of a single semiflexible filament by its surrounding may be modeled by a screening of the hydrodynamic self-interaction.

For the following it is important to realize that there is a profound difference between the hydrodynamic interaction of flexible polymers and semiflexible polymers in semidilute solutions. A flexible polymer is coiled and is thus far more likely to interact with itself than with surrounding polymers. On the other hand, semiflexible filaments like actin are much more stretched and strongly interact with each other even at quite low concentrations (Figure 3). As the scattering vector $\boldsymbol{k}$ in our experiments is large enough in magnitude to resolve single actin filaments (see Eq. (1.1)), we are interested in the dynamics of a single filament and use a mean field approximation to model the hydrodynamic interaction with the surrounding, i.e. we introduce a screening of the hydrodynamic self-interaction along a single polymer. This is achieved by use of the (preaveraged) screened transverse mobility matrix,

$$
\boldsymbol{H}_{\perp}(\boldsymbol{r})=\frac{e^{-r / \Lambda}}{8 \pi \eta r}\left(\mathbf{1}-\frac{|\boldsymbol{r}\rangle\langle\boldsymbol{r}|}{r^{2}}\right),
$$

where $\boldsymbol{r}:=\boldsymbol{r}_{s}-\boldsymbol{r}_{s^{\prime}}, \Lambda$ is the screening length and $\eta$ the solvent viscosity. Beyond this length $\Lambda$, the hydro- dynamic self-interaction of a filament is weak and correlations decay rapidly. The explicit form of the projector is a consequence of modelling the actin filament as a straight rod on length scales smaller than $\Lambda$ with respect to the hydrodynamic interaction. This should be a good approximation for $\xi \ll L_{p}$.

To simplify the calculation we use a kinetic coefficient $\zeta_{\perp}^{-1}$

$$
\left(\zeta_{\perp} / L\right)^{-1}=\frac{\log \Lambda / a}{4 \pi \eta}
$$

in place of the tensor $\boldsymbol{H}_{\perp} . \zeta_{\perp}$ is a renormalized friction, obtained by taking the terms in parentheses in Eq. (2.3) out of the integral and averaging over all segment positions $s$ for a rigid rod of diameter $a$. Replacing the mobility matrix by a simple coefficient amounts to setting the effective friction for all modes equal to the friction of the dominant mode of wavelength $\Lambda$. In this approximation interactions between different modes are neglected and the mobility for the very short wavelength modes is supposedly slightly overestimated. The latter should not profit as much by correlations over distances larger than their wavelength, as is implied by Eq. (2.4). This approximation will allow for a simple expression [Eq. 2.8) below] for the structure factor and at the same time captures the main effect of the hydrodynamic self-interaction of the single polymers as well as their mutual interaction. A theoretical basis for the above ansatz was already worked out by Muthukumar and Edwards [18] and will be explained in more detail in a forthcoming paper [20].

$\Lambda, a$ and the persistence length $L_{p}$ are the three characteristic length scales in terms of which the dynamics of a semiflexible polymer in semidilute solution is characterized. The microscopic cutoff parameter $a$ takes care of the finite thickness of the filament. Its value may be determined experimentally by the QELS method as described in the next subsection. We suppose that the hydrodynamic screening length $\Lambda$ may be identified (up to a numerical factor) with the mesh size $\xi$ of the actin network,

$$
\Lambda \simeq \xi \sim c_{a}^{-\frac{1}{2}}
$$

(Basically the same relation $\Lambda \sim c^{-1 / 2}$ can be derived within the effective medium approach for rods $[18$.) It is through Eq. (2.6) that the actin concentration $c_{a}$ ultimately enters Eq. (2.8) for the structure factor. The scaling law for the mesh size $\xi$ in Eq. (2.6) should be valid in the semidilute regime, when $\xi \leq L_{p}$ and the solution appears as a random network of almost rodlike segments. This was indeed confirmed experimentally [19]. The dimensionless quantity $\Lambda / a$, which could be called the "hydrodynamic aspect ratio" of the polymer, is actually the only characteristic parameter of the filament entering expression (2.8) for the structure factor besides the persistence length $L_{p}$ (cf. Eq. (2.9) below). 
Detailed calculations [20] show that the relaxation time of the $p^{t h}$ mode is $\tau_{p}=\frac{\zeta_{\perp} / L}{\kappa}\left(\frac{L}{\pi p}\right)^{4}$, as one would guess from dimensional analysis, and that in the intermediate time regime ${ }^{1}$,

$$
\left(k L_{p}\right)^{-4 / 3} \gamma_{k}^{-1} \ll t \ll \tau_{1},
$$

the structure factor factor is to a good approximation given by a stretched exponential

$$
g(\boldsymbol{k}, t)=g(\boldsymbol{k}, 0) \exp \left(-\frac{2 \Gamma(1 / 4)}{9 \pi}\left(\gamma_{k} t\right)^{3 / 4}\right) .
$$

The decay rate,

$$
\gamma_{k}=\frac{k_{B} T}{\zeta_{\perp} / L} k^{8 / 3} L_{p}^{-1 / 3},
$$

depends on the filament stiffness through the persistence length $L_{p}$ and on the actin concentration $c_{a}$ (or the mesh size) through the renormalized friction coefficient $\zeta_{\perp}$. It should be compared with the initial decay rate given below in Eq. (2.11) for the dilute case. Both are expected to play an important role in many dynamical problems, i.e. they are supposed to constitute the characteristic time scales of motion.

Some remarks have to be made on the derivation and the domain of validity of Eq. (2.8). First of all it is important to realize that the local motion of the monomers is neither strictly isotropic (like in the random coil limit) nor strictly transverse to the mean contour (as was assumed in Eq. (2.4)). The averaging over the different orientations can be performed in both limits [20]. Upon neglecting the anisotropy of the local segment motion, one obtains the simple stretched exponential from of the dynamic structure factor, Eq. (2.8). In the opposite limit - i.e. for local segment motion strictly transverse with respect to the mean contour - averaging over the different orientations is performed after calculating the anisotropic dynamic structure factor for an individual macromolecule, whose mean orientation and center of mass position is assumed to be fixed in space and time. In our case this is realized by the time scale separation mentioned above and by the fact that the macromolecules are imbedded in a network. It is found that the resulting dynamic structure factor is very similar to the stretched exponential form obtained by neglecting the anisotropy of the segment motion in the averaging procedure. We propose to use the stretched exponential form also in the intermediate regime, where the segment motion is neither strictly transverse nor isotropic. This allows for a simple

\footnotetext{
${ }^{1}$ The second condition is actually modified by entanglement. This may indeed be exploited in future high precision measurements, as is explained in 20], but is of minor importance here.
}

fit to the experimental data and has the advantage that the relevant physical mechanisms are not obscured by complicated numerical analysis. But, as a consequence of the approximations made, the numerical value of the prefactor in the exponential of Eq. (2.8) should be taken with some precaution. Unfortunately, the value obtained for the persistence length by Eq. (2.8) is very sensitive to this prefactor as well as to all the experimental parameters entering the exponent because the persistence length enters the expression for the decay rate, Eq. (2.9), as $L_{p}^{1 / 3}$. In addition the actual prefactor in the scaling law, Eq. (2.6), for $\Lambda$ is not known. Hence the method described here is not capable of producing very accurate absolute values for the bending modulus so far. On the other hand, relative changes in the stiffness, which may be caused by diverse chemical or physical mechanisms are readily detected.

\section{B. Initial Slope of the Dynamic Structure Factor}

Although the dynamic structure factor $g(\boldsymbol{k}, t)$ is a complicated object, and several physical assumptions and approximations enter its explicit calculation, its initial slope may be evaluated exactly in the case of Eq. (1.1). The general scheme of computation may be found in Ref. 16] (see also Ref. [21] for a summary of predictions derived from various semiflexible models). In the semiflexible case caution has to be paid to the rigid constraint of constant contour length. It causes an effective reduction of the degrees of freedom of the local quasi-free Brownian fluctuations about the equilibrium configuration, which determine the initial slope for large scattering vectors and moderate chain stiffness. (For very stiff molecules the time regime for these quasi-free fluctuations diminishes [6], but actin is far from this limit as can be inferred from a comparison of the characteristic time scales $\tau_{p}, \gamma_{k}$ and $\gamma_{k}^{(0)}$.) For the detailed calculation of the initial decay rate,

$$
\gamma_{k}^{(0)}:=-\left.\frac{d}{d t} \log g(\boldsymbol{k}, t)\right|_{t=0},
$$

for the case of a semiflexible polymer in semidilute solution we refer the reader to Ref. [20]. Here we give the asymptotic result for a dilute solution and for scattering wave vectors $\boldsymbol{k}$ of modulus $k$ larger than the inverse persistence length $L_{p}^{-1}$ but much smaller than the inverse of the microscopic cutoff length $a$ introduced in Eq. (2.5): 


$$
\gamma_{k}^{(0)}=\frac{k_{B} T}{6 \pi^{2} \eta} k^{3}\left(\frac{5}{6}-\log k a\right) .
$$

The deviation from ideal scaling is rather weak, hence it is useful to express Eq. 2.11) as a "quasi-scaling law" $\gamma_{k}^{(0)} \sim k^{z(k)}$ with an effective dynamic exponent (Figure 4) given by

$$
z(k)=3 \frac{6 \log k a-3}{6 \log k a-5} .
$$

It is quite striking that the microscopic cutoff $a$ appears here. Hydrodynamic screening does not substantially alter the result of Eq. (2.11) in the large wave vector regime, but flattens the increase of $\gamma_{k}^{(0)} / k^{3}$ for small wave vectors. However, the theory is not valid for small scattering vectors, because scattering vectors smaller than the inverse mesh size $\xi^{-1}$ do not resolve single filaments but average over several molecules. Moreover, as a concequence of local fluctuations in the mesh size there is scattering in the experimental data in the crossover region $k \approx \xi^{-1}$.

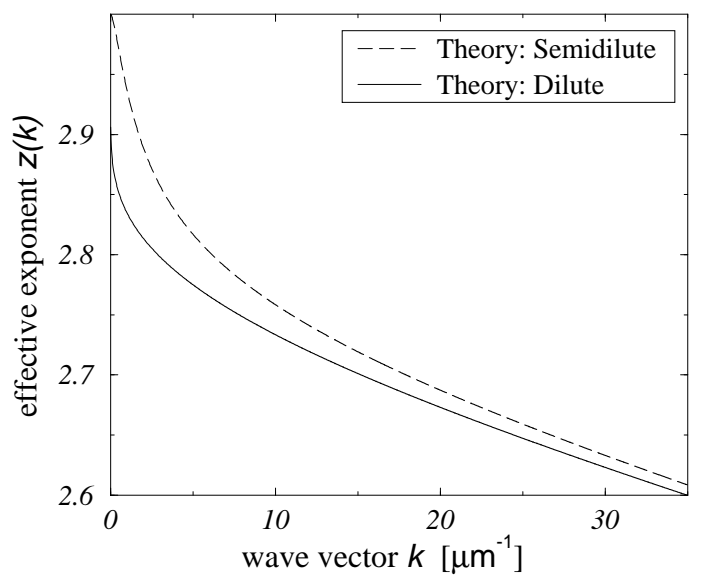

FIG. 4. Effective exponent $z(k)$ of the "quasi-scaling" law for the initial decay rate of the dynamic structure factor of actin. $z$ was predicted to be a universal number, $z=3$, for all flexible polymers by classical scale invariant models. This was never observed experimentally. We argue that the (intrinsic) semiflexibility of all real polymers is responsible for the discrepancy. The dashed line was computed for a semidilute solution of actin filaments $\left(c_{a}=0.16 \frac{\mathrm{mg}}{\mathrm{ml}}\right)$. The solid line corresponds to the dilute case, Eq. (2.12). In the typical $k$ intervall probed by QELS $\left(5 \sim 30 \mu \mathrm{m}^{-1}\right) z(k) \approx 2.7$ for actin.

Figure 5 shows a comparison of the computed initial decay rate $\gamma_{k}^{(0)}$ for a solution of $0.16 \frac{\mathrm{mg}}{\mathrm{ml}}$ actin (dashed line) with previously measured data [22,19]. A simple least square fit of Eq. (2.11) to the data gives $a=5.4$ nm. $a$ represents an effective hydrodynamic diameter of the filament to be compared with two times the cross sectional radius of gyration $r_{\perp}^{g}$. The remarkable agreement of $a$ with the value of $2 r_{\perp}^{g^{\perp}}=5.16 \pm 0.3 \mathrm{~nm}$ determined by different methods [24,25] strongly supports the above ideas.

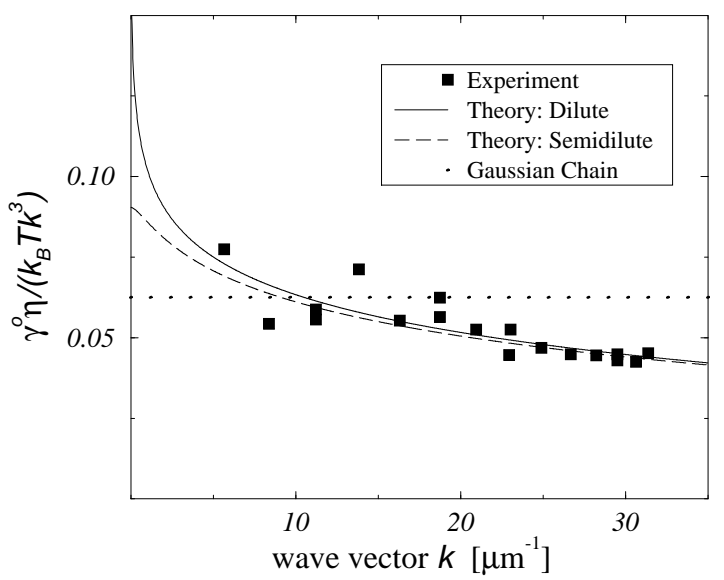

FIG. 5. Correction to the classical prediction $\gamma_{k}^{(0)} \sim k^{3}$ for the initial decay rate of the dynamic structure factor. The theoretical predictions for dilute solutions (solid line) and semidilute solutions (dashed line) are compared with experimental data of Schmidt [22,19. Also included is the prediction for gaussian chains from Ref. [16]. The experimental data and the dashed line both correspond to the same actin concentration $c_{a}=0.16 \frac{\mathrm{mg}}{\mathrm{ml}}$. The positions of the theoretical curves depend on the effective hydrodynamic diameter $a$ of the filament [see Eq. 2.11]. It was used as fit parameter and found to be $a=5.4 \mathrm{~nm}$.

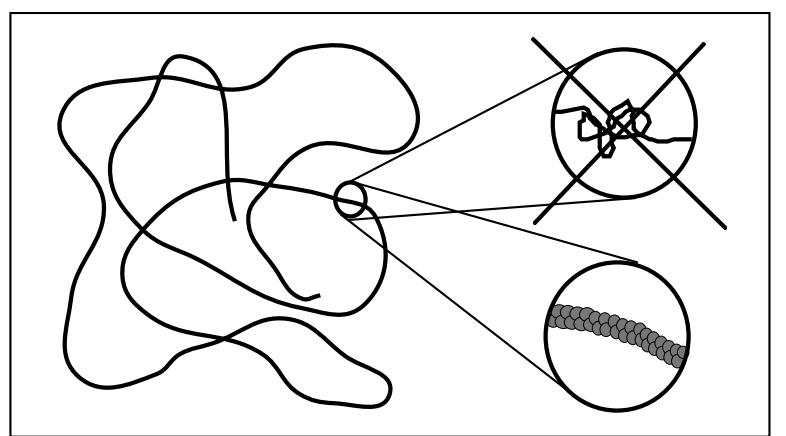

FIG. 6. A real polymer is not a fractal (i.e. not a gaussian chain, as often assumed for computational convenience) but rodlike on small scales. This affects the dynamical properties of polymers.

Finally we would like to note that in classical neutron scattering experiments with more or less flexible synthetic polymers the ratio of wavelength to persistence length $\lambda / L_{p}$ is not very different from what is encountered in light scattering from large biomolecules. So one expects similar results in both cases. It is well known [23,16] that there is sometimes poor agreement between the experiments with synthetic polymers and the classical theoretical predictions for the initial slope of $g(\boldsymbol{k}, t)$ 
derived from scale invariant models. The excellent agreement achieved now with actin networks provides strong evidence that the usual scale invariant polymer models are not capable of describing quantitatively the dynamic properties of real polymers, which, after all, are semiflexible at heart (Figure 6).

\section{MATERIALS AND METHODS}

Actin was prepared from rabbit muscles according to Pardee and Spudich 26 with an additional gel filtration step as suggested by MacLean-Fletcher and Pollard 27 using a Sephacryl S-300 HR column. Tropomyosin/Troponin were prepared from the residue of rabbit muscle acetone powder left after the actin extraction [28], and separated into tropomyosin and troponin by hydroxyl apatite column chromatography [29]. The purity of the proteins was checked by SDS polyacrylamide gel electrophoresis [30] stained with Commassie Blue, and estimated to be of at least 95\% purity. Actin was tested for its ability to polymerize by low shear viscometry with the falling ball capillary apparatus as described by Pollard and Cooper [31], and by fluorescence increase of 5\% NBD-labeled actin [32]. Functionality of tropomyosin/troponin in the presence and absence of $\mathrm{Ca}^{++}$was characterized by an actin binding test: they were added to F-actin in varying concentration ratios, centrifuged at $100000 \mathrm{~g}$ for $1 \mathrm{~h}$, and analyzed by SDS gel electrophoresis.

Actin was stored in a buffer containing $2 \mathrm{mM}$ imidazole, $0.2 \mathrm{mM}$ ATP, $0.2 \mathrm{mM}$ DTT, $0.2 \mathrm{mM} \mathrm{CaCl}_{2}$ and 0.05 vol. $\% \mathrm{NaN}_{3}$. For the polymerization of actin, a buffer with $2 \mathrm{mM}$ imidazole, $0.5 \mathrm{mM}$ ATP, $2 \mathrm{mM}$ $\mathrm{MgCl}_{2}, 100 \mathrm{mM} \mathrm{KCl}, 0.2 \mathrm{mM}$ DTT and $0.2 \mathrm{mM} \mathrm{CaCl}$ was used. The buffers where adjusted to a $\mathrm{pH}$ of 7.4. For the experiment without $\mathrm{Ca}^{++}$the $\mathrm{CaCl}_{2}$ was left out and $1 \mathrm{mM}$ EGTA was added. The molar ratio of actin:tropomyosin:troponin was 7:1:1.

For QELS measurements, all solutions were freed from dust by sterile filtration, mixed, and stored overnight at $4^{\circ} \mathrm{C}$ to achieve an equilibrium polymerisation state. Samples to be inspected by electron microscopy were adsorbed on glow discharged carbon coated formvar films on copper grids, and negatively stained with uranyl acetate.

The experimental setup for QELS has been described in detail previously 19,33 . We used the correlator ALV 3000 (ALV Langen) with 1024 linear channels to calculate the dynamic structure factor. The light source was an Innova 70-4 argon-ion laser from Coherent with 200 $\mathrm{mW}$ for the $488 \mathrm{~nm}$ line.

\section{RESULTS}

We now turn to a discussion of the experimental data and their analysis in terms of the theory described above.

\section{Stretched Exponential}

Figure 7 shows a fit of the theoretical dynamic structure factor, Eq. 2.8), to experimental data for a scattering angle of $90^{\circ}$ corresponding to $k=24.2 \mu \mathrm{m}^{-1}$. Note that there is only one free parameter, $\gamma_{k}$. An excellent fit of the experimental curves is obtained in the time domain $10^{-5} \sim 10^{-2} \mathrm{~s}$, for which the condition, Eq. (2.7), is approximately fulfilled (cf. Footnote 1), whereas a simple exponential decay is clearly ruled out. Hence the theory is well suited to interpret our data within the present experimental accuracy.

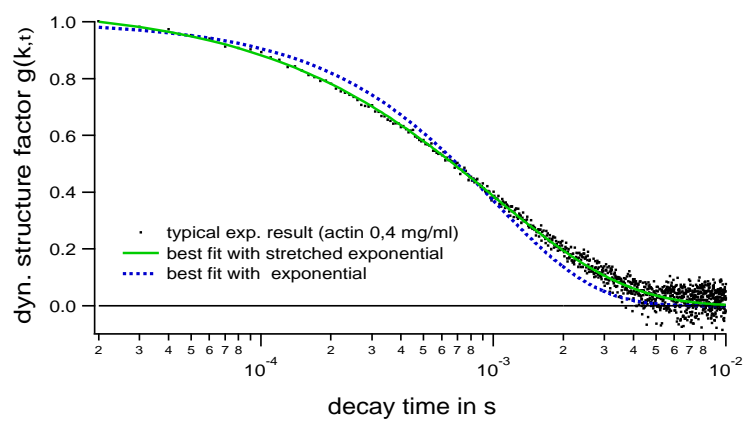

FIG. 7. Fit of theoretical dynamic structure factor Eq. (2.8) to experimental data for $k=24.2 \mu \mathrm{m}^{-1}$. Clearly Eq. (2.8) describes very well the experimental situation in the time interval $10^{-5} \sim 10^{-2} \mathrm{~s}$, wheras the simple exponential fit is ruled out.

\section{Bending Modulus}

As pointed out in Section II A the decay rate $\gamma_{k}$, Eq. (2.9), is determined by the bending modulus $\kappa$ and also by the screening length $\Lambda$. In order to check the effect of the screening length on the decay rate, we measured the dynamic structure factor for various actin concentrations $c_{a}$. The screening length was taken to be equal to the mesh size $\xi$, which was found previously 19] to obey the scaling law $\xi[\mu \mathrm{m}]=0.35 \sqrt{\mathrm{c}_{\mathrm{a}}\left[\frac{\mathrm{mg}}{\mathrm{ml}}\right]}$. The results are shown in Figure 8. As discussed in Section IIB, the theory only applies to scattering vectors large enough to resolve single filaments. Scattering vectors $k$ smaller than the inverse mesh size $\xi^{-1}$ average over several filaments. Hence, the decay of the structure factor obeys Eq. 2.9) only for large scattering vectors $k$. The onset of the deviation may be taken as a lower bound for the mesh size.

Although the concentration was increased by a factor of 8 the derived values for the bending modulus $\kappa$ are 
fairly consistent. They agree within a standard deviation of $35 \%$ for the entire range of scattering vectors and concentrations. However, for a single scattering vector and fixed concentration the deviations are considerably smaller, e.g. for $k=24.2 \mu \mathrm{m}^{-1}\left(90^{\circ}\right)$ and $0.4 \frac{\mathrm{mg}}{\mathrm{ml}}$ the data are reproducible within $5 \%$. Considering the discussion at the end of Section IIA and the dependence of the value of the bending modulus $\kappa$ derived from Eq. 2.8 on the hydrodynamic aspect ratio $\Lambda / a-$ and hence on our choice of the screening length $\Lambda$ - we are presently not able to determine an accurate absolute value for $\kappa$. (An average value of $9.5 \cdot 10^{-27} \mathrm{Jm}$ was obtained for $\kappa$.) On the other hand, relative changes in the stiffness can be resolved rather precisely.

Methods to determine $\Lambda$ experimentally are discussed in Section V.

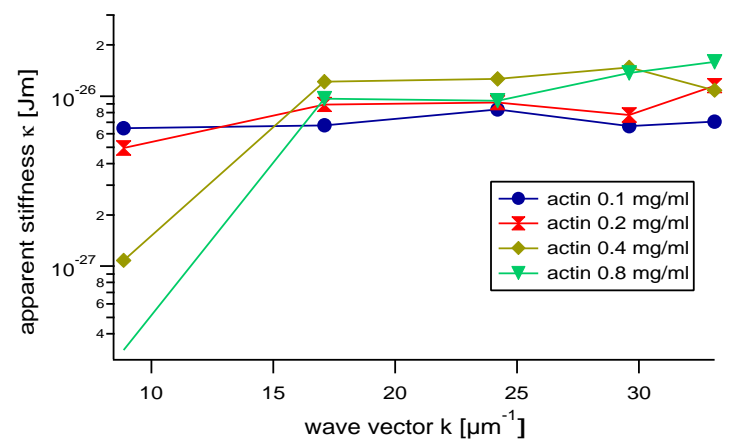

FIG. 8. Values for the bending modulus $\kappa$ obtained for various actin concentrations $c_{a}$ in the semidilute regime using Eq. (2.8) and $\Lambda=\xi$. Only large wave vectors $k \gg \xi^{-1}$ resolve single filaments and are accessible to our theory.

\section{Effect of Tm/Tn Binding}

Figure 9 shows the effect of the tropomyosin/troponin complex $(\mathrm{Tm} / \mathrm{Tn})$ on the decay of the dynamic structure factor for actin solutions with a concentration of $c_{a}=0.3 \frac{\mathrm{mg}}{\mathrm{ml}}(\simeq 7.1 \mu \mathrm{M})$ at $10^{\circ} \mathrm{C}$. Since $\mathrm{Ca}^{++}$is known to regulate the coupling of the Tm/Tn-complex to actin, experiments were performed with and without $\mathrm{Ca}^{++}$. Figure 9 clearly shows the appreciable decrease of the decay rate in the presence of $\mathrm{Tm} / \mathrm{Tn}$. The reduction of $\mathrm{Ca}^{++}$ appears to decrease the stiffness slightly but the effect is too weak to be considered significant with the present accuracy of measurement. The experiments were repeated several times with different actin preparations and at two different temperatures $\left(10^{\circ} \mathrm{C}\right.$ and $\left.25^{\circ} \mathrm{C}\right)$ and the same absolute value of $\kappa$ as well as the same degree of stiffening by Tm/Tn was always observed. The results of the measurements at $10^{\circ} \mathrm{C}$ are summarized in Table $\mathbb{I}$

\begin{tabular}{|l|r|r|r}
\hline \hline scattering vector $k\left[\mu \mathrm{m}^{-1}\right]$ & 17.1 & 24.2 & 29.6 \\
\hline$\kappa$ for actin $\left[10^{-27} \mathrm{Jm}\right]$ & 9.9 & 8.7 & 8.6 \\
\hline$\kappa$ for actin $+\mathrm{Tm} / \mathrm{Tn}-\mathrm{Ca}^{++}$ & 11.4 & 12.9 & 14.7 \\
\hline$\kappa$ for actin $+\mathrm{Tm} / \mathrm{Tn}+\mathrm{Ca}^{++}$ & 12.1 & 15.9 & 13.9 \\
\hline \hline
\end{tabular}

TABLE I. Summary of values obtained for the bending modulus $\kappa[\mathrm{Jm}]$ of actin in the presence of $\mathrm{Tm} / \mathrm{Tn}$ with and without $\mathrm{Ca}^{++}$at different scattering angles in comparison to pure actin.

To exclude preparation artifacts and in order to check whether Tm/Tn has some effect on the mesh size, which would in turn affect the hydrodynamic screening length $\Lambda$ and thus the derived value of $\kappa$, the actin network was examined by negative-staining EM for all samples. We could not observe an effect of Tm/Tn on the network structure. In summary, we find that in the presence of $\mathrm{Ca}^{++} \mathrm{Tm} / \mathrm{Tn}$ causes an increase of the bending modulus of F-actin by about $50 \%$.

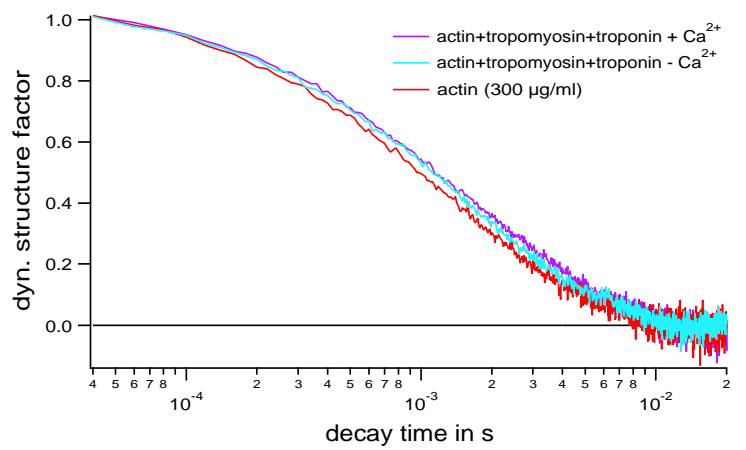

FIG. 9. Effect of Tm/Tn binding with $\mathrm{Ca}^{++}(0.2 \mathrm{mM}$ uppermost curve) and without $\mathrm{Ca}^{++}$(closely below) in comparison with the pure actin sample (lower curve) observed at a scattering angle of $90^{\circ}$, temperature $T=10^{\circ} \mathrm{C}$ and actin concentration $c_{a}=0.3 \frac{\mathrm{mg}}{\mathrm{ml}}$.

\section{GENERAL DISCUSSION}

The present analysis of the dynamic structure factor of semidilute entangled actin solutions shows that QELS is a reliable tool for the study of the internal dynamics of semiflexible polymers. However, as may be seen from Eq. (2.9), the interpretation of experimental data is not entirely straightforward. The expression for the dynamic structure factor (2.8) is ambiguous with respect to the microscopic source of an observed change in the decay rate. Changes may be caused by variations in the intrinsic stiffness of the filament as well as by variations of the mesh size of the network, which in turn affect the screening length $\Lambda$. As experiments with $\alpha$-actinin show [34], even a local change in the mesh size, as caused by such 
crosslinking proteins, results in a corresponding change of the decay rate over the whole range of scattering vectors. This is exactly what one would expect from Eq. (2.9), if the main contribution to the scattered light is attributed to the crosslinked clusters (seen in EMs), which contain most of the filaments.

The main uncertainty in the quantitative predictions of the QELS method described above presently arises from the uncertainty in the absolute value of the hydrodynamic screening length $\Lambda$. Setting it equal to the mesh size seems to be reasonable, but perhaps one could do better. There are various methods to determine $\Lambda$. The QELS method itself as described above could be used, if $\kappa$ was known. Direct information on $\Lambda$ should be provided by the autocorrelation of a labeled filament in solution, which may be investigated by fluorescence microscopy in the case of actin. Another approach exploits reptation. In the same manner as the transversal friction, Eq. (2.5), one may introduce the kinetic coefficient of the longitudinal center of mass diffusion of semiflexible filaments in entagled networks $\zeta_{\|}=2 \pi \eta L / \log (\Lambda / a)$.

There are also effects from the center of mass and rotational motion, which we have neglected so far. Because of the polydispersity of the actin solution, some filaments shorter than the mesh size $(L<\xi)$ that are free to rotate and diffuse are always present. Their hydrodynamic correlation is not disturbed, nor are they hindered by entanglement. The center of mass and rotational dynamics of those short filaments may to a good approximation be described by the theory for rigid rod molecules 16] (for a more complete treatment including bending motions see [6]). For the time being, the accuracy of the experimental data does not allow for a quantitative analysis of this small effect.

Finally we want to compare our findings with results obtained by the fluorescence technique. The two methods can be considered complementary: They probe adjacent wavevector and time regimes. The fluorescence technique is more direct. QELS, on the other hand, avoids problems associated with potential perturbations from fluorescence markers (for the case of actin and phalloidin see Ref. [24]) and gives much better statistics. For the time being, a significant discrepancy remains concerning the effect of $\mathrm{Tm} / \mathrm{Tn}$. Tm/Tn was seen to cause a softening of the filaments with the fluorescence technique [- This contradicts the QELS data given above as well as some recent direct measurements 35,36]. We do not have a fully convincing explanation for this discrepancy. A stiffening of actin by $\mathrm{Tm} / \mathrm{Tn}$ binding seems very probable, since other proteins binding along actin filaments are also known to enhance the stiffness of the filaments, e.g. talin [37], a protein involved in the membrane binding of actin in cells.

However, one may also think of a more intriguing scenario in terms of torsional modes and other nonlinear or higher order derivative contributions to the configurational energy of the filament. No thorough theoretical treatment of all those possible contributions has been given so far, and it is not known, which of them are actually relevant. A first principles analysis of the various linear modes of actin (in vacuo) has only been published very recently [38]. Especially for F-actin local torsional modes and additional "groove swinging" and axial slipping motions are thought to be important because of its double-stranded structure. Very strong evidence for local torsional excitations was provided by high resolution electron microscopy studies [24] showing that the twist angle is not fixed but may fluctuate by $\pm 10^{\circ}$. Torsional fluctuations of some $100 \mathrm{~nm}$ length have been identified. The presence of torsional modes may affect the dynamic structure factor in two ways: First, they cause "crankshaft" motions of bent chains and thus provide an additional mechanism contributing to the time decay of the correlation function. Second, they could lead to a scale dependent renormalized bending stiffness of actin as was recently observed for the railway track model [39]. Though we did not see any systematic $k$ dependence of the bending modulus with the QELS method, such effects may well occur in other kregimes. The more "exotic" motions of actin are of great biological interest, since they could play an active role in the conformational changes of the actin-myosin-complex during ATP-cleavage. It is hoped that future QELS studies with enhanced precision and larger ranges of scattering vectors and measurement times will help to clarify this important point.

Acknowledgment: This work was supported by the Deutsche Forschungsgemeinschaft (Sa 246/24-1 and Fr 850-2) and in part by the National Science Foundation under Grant No PHY 89-04035. One of the authors (E. Sackmann) gratefully acknowledges the hospitality encountered during his stay at the Institute for Theoretical Physics, UCSB, under the directorship of J. Langer. We are grateful to Toni Maggs, Kurt Kremer and Joseph Käs for enlightening discussions and for making available unpublished results. We thank Irene Sprenger, who contributed to this work by making many EM-micrographs, and our biochemical laboratory staff for the preparation of the proteins.

[1] Janmey P.A.; Hvidt, S.; Käs J.; Lerche, D.; Maggs, A.; Sackmann, E.; Schliwa, M.; Stossel Th. P. J. Biol. Chem. 1994, 269/51, 32503.

[2] Müller, O.; Gaub, H. E.; Bärmann, M.; Sackmann, E. Macromolecules 1991, 24, 3111.

[3] Ziemann, F.; Rädler, J.; Sackmann E. Biophys. J. 1994, 66,2210 .

[4] Käs, J.; Strey, H.; Bärmann, M.; Sackmann, E. Europhys. Lett. 1993, 21 (8), 865. 
[5] Ott, A.; Magnasco, M.; Simon, A.; Libchaber, A. Phys. Rev. E 1993, 48, 3, 1642.

[6] Song, L., Kim, U-S., Wilcoxson, J., Schurr, J.M. Biopolymers 1991, 31, 547.

[7] Aragón, S.R. Macromolecules 1991, 24, 3451.

[8] Harnau, L., Winkler, R.G., Reineker, P. J. Chem. Phys. 1995, 102 (19), 7750.

[9] Aragón, S.R. and Pecora, R. Macromolecules 1985, 18, 1868.

[10] Harris, R.A. and Hearst J.E. J. Chem. Phys. 1966, 44, 2595. Harris, R.A. and Hearst J.E. and Beals, E. J. Chem. Phys. 1966, 45, 3106. Harris, R.A. and Hearst J.E. J. Chem. Phys. 1967, 46, 298.

[11] Lagowski, J.B., Noolandi, J., Nickel, B. J. Chem. Phys. 1991, 95 (2), 1266.

[12] Winkler, R.G., Reineker, P., Harnau, L. J. Chem. Phys. 1994, 101 (9), 8119.

[13] Maeda, T., Fujime, S. Macromolecules 1984, 17, 2381.

[14] Kratky, O.; Porod, G. Rec. Trav. Chim. 1949, 68, 1106.

[15] de Gennes, P.-G. Physics 1967, 3, 1, 37./ DuboisViolette, E.; de Gennes, P.-G. Physics 1967, 3, 1, 181.

[16] Doi, M.; Edwards, S. F. The theory of polymer dynamics. Clarendon Press: Oxford, 1986, 228.

[17] Farge, E.; Maggs, A. C. Macromolecules 1993, 26, 19, 5041.

[18] Muthukumar, M., Edwards, S.F. Macromolecules 1983, $16,1475$.

[19] Schmidt, C.; Bärmann, M.; Isenberg, G.; Sackmann, E. Macromolecules 1989, 22, 3638.

[20] Kroy, K.; Frey E. to be published.

[21] Schmidt, M. and Stockmayer, W.H. Macromolecules 1984, 17, 509.

[22] Schmidt, Ch; Doctoral Thesis, TU München 1988.
[23] de Gennes, P.-G. Scaling Concepts in Polymer Physics 1979. Cornell.

[24] Bremer, A. ; Milloing, R. C.; Sütterlin, R.; Engel, A.; Pollard, T. D.; Aebi, U.; J. Cell Biology, 1991, 115, 3, 689.

[25] Egelman, E. H.; Padrón, R. Nature 1984, 307, 56.

[26] Pardee, J.D.; Spudich, J. A. Methods Enzymol. 1982, 85, 164.

[27] MacLean-Fletcher, S.; Pollard, T. D. Biophys. Biochem. Res. Commun. 1980, 96, 18

[28] Spudich, J.A; Watt, S. J. Biol. Chem. 1971, 249, 4866.

[29] Eisenberg, E.; Kielley, W. W. J. Biol. Chem. 1974, 249, 4742 .

[30] Laemmli, U. K. Nature 1970, 227, 680.

[31] Pollard, T.D.; Cooper, J. A. Methods Enzymol. 1982, 85, 211.

[32] Detmers, P.; Weber, A.; Elzinga, M.; Stephens, R.E. J. Biol. Chem. 1981, 256, 99.

[33] Piekenbrock, Th.; Sackmann, E. Biopolymers 1992, 32, 1471.

[34] Götter, R. et al, to be published.

[35] Kojima, H., Ishijima, A., Yanagida, T., Proc. Natl. Acad. Sci. USA 1994, 91, 12962.

[36] Isambert, H., Veniers, P., Maggs, A., C., Fattoum, A., Kassab, R., Pantaloni, D., Carlier, M.-F., J. Bio. Chem. $1995270,11437$.

[37] Ruddies, R.; Goldmann, W. H.; Isenberg, G.; Sackmann, E. Eur. Biophys. J. 1993, 22, 309.

[38] Ben-Avraham, D. and Tirion, M. Biophys. J. 1995, 68 1231.

[39] Everaers, R.; Bundschuh, R.; Kremer, K. Europhys. Lett. 1995, 29, 263. 